\title{
NEW EXACT SOLUTIONS OF SPATIALLY AND TEMPORALLY VARYING REACTION-DIFFUSION EQUATIONS
}

\author{
NALINI JOSHI AND TEGAN MORRISON
}

\begin{abstract}
This paper considers reaction diffusion equations from a new point of view, by including spatiotemporal dependence in the source terms. We show for the first time that solutions are given in terms of the classical Painlevé transcendents. We consider reaction diffusion equations with cubic and quadratic source terms. A new feature of our analysis is that the coefficient functions are also solutions of differential equations, including the Painlevé equations. Special cases arise with elliptic functions as solutions. Additional solutions given in terms of equations that do not have the Painlevé property are also considered. Solutions are constructed using a Lie symmetry approach.
\end{abstract}

\section{INTRODUCTION}

This paper presents solutions to the following two reaction diffusion equations

$$
\begin{gathered}
u_{t}=u_{x x}+q_{0}(x, t) u-u^{2}, \\
u_{t}=u_{x x}+q_{1}(x, t) u+q_{2}(x, t) u^{2}-u^{3} .
\end{gathered}
$$

The coefficient functions $q_{i}(x, t), i=0,1,2$, are not specified explicitly at the outset, but are chosen to satisfy particular equations which enable exact solution of equations (1.1) and (1.2). To the authors' knowledge solutions of reaction diffusion equations of this form have not been previously studied. The motivating equations for the current work are the standard reaction diffusion equations with quadratic and cubic source terms. These equations are ubiquitous in biological and physical systems (see $[5,8,9])$ and take the following form: Fisher's equation

$$
u_{t}=u_{x x}+u(1-u) \text {, }
$$

and, the Nagumo and Huxley-type equations respectively,

$$
u_{t}=u_{x x}+u(1-u)(u-a), \quad u_{t}=u_{x x}+u^{2}(1-u),
$$

where $a$ is constant. By generalising the Fisher and Nagumo equations to include variable coefficients we arrive at equations (1.1) and (1.2), which are the focus of this paper. Equations of Huxley-type with spatial heterogeneity included in the source terms are considered in [4].

Solutions of the reaction diffusion equations (1.1) and (1.2) are constructed by exploiting their invariance under Lie groups of point symmetries. We find that these solutions can only be constructed if the coefficient functions $q_{i}(x, t)$ satisfy particular partial differential equations. In this case, both equations (1.1) and (1.2)

Date: February 26, 2008.

Dedicated to the memory of NJ's friend, teacher, and mentor Martin David Kruskal (19252006). NJ's and TM's research was supported by the Australian Research Council Discovery Program grant \#DP0664624 and an Australian Postgraduate Award. 
are found to admit a three parameter group of point symmetries. Furthermore, the equations for $q_{i}(x, t)$ also admit a three parameter group of symmetries. These symmetries are used to reduce both sets of partial differential equations to ordinary differential equations. We find that the reduced ordinary differential equations corresponding to (1.1) are explicitly linked with equations of the form

$$
y^{\prime \prime}(\lambda)=6 y^{2}+f(\lambda),
$$

which have the Painlevé property if $f(\lambda)$ is linear. Similarly, we find that the reduced ordinary differential equations corresponding to (1.2) are explicitly linked with equations of the form

$$
y^{\prime \prime}(\lambda)=2 y^{3}+g(\lambda) y+h(\lambda),
$$

which have the Painlevé property if $g(\lambda)$ is linear and $h(\lambda)$ is constant. Thus, we find exact solutions to reaction diffusion equations, which are not usually integrable, in terms of associated integrable ordinary differential equations.

Solutions to equations (1.1) and (1.2) are given in terms of classical Painlevé transcendents. The Painlevé equations are six non-linear second order differential equations with the special (Painlevé) property that all solutions are single valued around moveable singularities. The generic solutions to these equations cannot be expressed in terms of standard functions, thus they define new transcendental functions. Of interest here are the first and second Painlevé equations which are

$$
\mathrm{P}_{\mathrm{I}}: \quad y^{\prime \prime}=6 y^{2}+\lambda, \quad \mathrm{P}_{\mathrm{II}}: \quad y^{\prime \prime}=2 y^{3}+\lambda y+\alpha,
$$

where $y$ is a function of $\lambda$ and $\alpha$ is a constant. These equations are integrable examples of equations (1.3) and (1.4) respectively and thus they are explicitly linked with the exact solutions of equations (1.1) and (1.2). The recognition of the connection between Painlevé equations and variable coefficient reaction diffusion equations of the form (1.1) and (1.2) is believed to be new.

Solutions to equations (1.1) and (1.2) are also given in terms of Weierstrass and Jacobi elliptic functions. These functions solve the autonomous forms of equations (1.3) and (1.4) respectively. In [1], travelling wave solutions to Fisher's equation are given in terms of Weierstrass elliptic functions. These solutions are obtained by demanding that the reduced ordinary differential equation passes the Painlevé test (see [7]). This restricts the wave speed to a particular value. The solutions that we construct for the generalised Fisher type equation (1.1) include this as a special case. Two examples of investigations of reaction diffusion equations which have found Jacobi elliptic function solutions are: [4], where a spatial dependence is included in a Huxley-type equation, and [6], where reaction diffusion equations with non-linear diffusion are considered. In both cases the solutions are constructed using non-classical symmetry methods. The simpler, classical symmetry approach followed in this paper produces not only these elliptic function solutions, but also the Painlevé transcendent solutions. The freedom that the coefficient functions $q_{i}(x, t)$ afford to equations (1.1) and (1.2) makes them most amenable to study.

\section{Symmetry Analysis}

Symmetry analysis provides a systematic way to construct solutions to partial differential equations. We follow the classical approach, which determines the forms 
of $X(x, t, u), T(x, t, u)$, and $U(x, t, u)$ for which the equation is invariant under the point transformations

$$
x_{1}=x+\varepsilon X(x, t, u), \quad t_{1}=t+\varepsilon T(x, t, u), \quad u_{1}=u+\varepsilon U(x, t, u), \quad \varepsilon \ll 1 .
$$

These point transformations form a group with generator

$$
\Gamma=X(x, t, u) \frac{\partial}{\partial x}+T(x, t, u) \frac{\partial}{\partial t}+U(x, t, u) \frac{\partial}{\partial u},
$$

and the invariants of the group, denoted $F$, are solutions of the equation $\Gamma F=0$. These invariants are used to construct similarity solutions of the partial differential equations, in terms of solutions to ordinary differential equations (see [3]).

2.1. Quadratic Case. We will refer to the function $q_{0}(x, t)$ as $q(x, t)$ in all discussion of equation (1.1). The partial differential equation (1.1) has no (non-trivial) classical symmetries if $q(x, t)$ is free. Fix $q(x, t)$ as a solution to following partial differential equation

$$
\frac{\partial q}{\partial t}=\frac{\partial^{2} q}{\partial x^{2}}+\frac{1}{2} q^{2}+k(x, t)
$$

where $k(x, t)$ is as follows

$$
\left(c_{1}+\frac{c_{3} x}{2}\right) \frac{\partial k}{\partial x}+\left(c_{2}+c_{3} t\right) \frac{\partial k}{\partial t}+2 c_{3} k=0 .
$$

Then, equation (1.1) is invariant under a three parameter Lie group of point symmetries with infinitesimal group operator (2.1) where

$$
\begin{aligned}
X(x, t, u) & =c_{1}+\frac{c_{3} x}{2}, \quad T(x, t, u)=c_{2}+c_{3} t, \\
U(x, t, u) & =\frac{1}{2}\left\{\left(c_{1}+\frac{c_{3} x}{2}\right) \frac{\partial q}{\partial x}+\left(c_{2}+c_{3} t\right) \frac{\partial q}{\partial t}\right\}-c_{3}\left(u-\frac{q}{2}\right),
\end{aligned}
$$

and $c_{1}, c_{2}, c_{3}$ are arbitrary parameters.

The partial differential equation (2.2) for $q(x, t)$ is also invariant under a three parameter Lie group of point symmetries if $k(x, t)$ satisfies (2.3) with $c_{i} \mapsto c_{i+3}$, $i=1,2,3$. In this case

$$
X(x, t, q)=c_{4}+\frac{c_{6} x}{2}, \quad T(x, t, q)=c_{5}+c_{6} t, \quad Q(x, t, q)=-c_{6} q,
$$

for arbitrary parameters $c_{4}, c_{5}, c_{6}$. Thus each of the similarity reductions of the independent variables valid for $u(x, t)$ are also valid for $q(x, t)$. The dependent variable terms in equation (2.2) for $q(x, t)$ come directly from equation (1.1) with $u(x, t)=q(x, t) / 2$. This relationship is captured in each reduction of the dependent variable $u(x, t)$, as follows in Table 1 .

We explore three possible reductions for each of $u(x, t)$ and $q(x, t)$ which gives rise to a total of nine possible cases. The reductions come from different linear combinations of the group operators associated with each constant $c_{1}, c_{2}, c_{3}$ in (2.4). The three reductions are (1) Stationary $c_{1}=c_{3}=0, c_{2}=1$; (2) Travelling Wave $c_{1}=c, c$ constant, $c_{2}=1, c_{3}=0$; (3) Boltzmann transformation $c_{1}=c_{2}=0$, $c_{3}=1$. These map the independent variables $(x, t)$ to the following new variables:

$$
\begin{aligned}
& \text { (1) } x=x(t \text { constant }) \text {, } \\
& \text { (2) } z=x-c t \text {, } \\
& \text { (3) } \zeta=\frac{x}{t^{1 / 2}} \text {, }
\end{aligned}
$$


respectively. The corresponding reductions of the dependent variable $u(x, t)$ are easily calculated from the characteristic equation associated with the group operator for each form of (2.4). Similarly, the reduced form of $k(x, t)$ follows from (2.3). Let the nine cases be numbered $(i j)$, where $u(x, t)$ is reduced according to $(i), i=1,2,3$ as above $(2.5)$, and $q(x, t)$ is reduced according to $(j), j=1,2,3$. These cases are summarised below in Table 1, with reference to equations in Table 2.

Table 1: Summary of reductions of equation (1.1)

\begin{tabular}{ccccc}
\hline Case & $u(x, t)$ & $\eta(*)$ equation & $q(*)$ equation & $k(*)$ \\
\hline \hline$(11)$ & $q(x) / 2+\eta(x)$ & $A_{1}$ & $B_{1}$ & $k(x)$ \\
$(12)$ & $q(z) / 2+\eta(x)$ & $A_{1}$ & $B_{2}$ & constant \\
$(13)$ & $q(\zeta) /(2 t)+\eta(x)$ & $A_{1}$ & $B_{3}$ & 0 \\
\hline$(21)$ & $q(x) / 2+\eta(z)$ & $A_{2}$ & $B_{1}$ & constant \\
$(22)$ & $q(z) / 2+\eta(z)$ & $A_{2}$ & $B_{2}$ & $k(z)$ \\
$(23)$ & $q(\zeta) /(2 t)+\eta(z)$ & $A_{2}$ & $B_{3}$ & 0 \\
\hline$(31)$ & $q(x) / 2+\eta(\zeta)$ & $A_{3}$ & $B_{1}$ & 0 \\
$(32)$ & $q(z) / 2+\eta(\zeta)$ & $A_{3}$ & $B_{2}$ & 0 \\
$(33)$ & $(q(\zeta) / 2+\eta(\zeta)) / t$ & $A_{3}$ & $B_{3}$ & $k(\zeta) / t^{2}$ \\
\hline \hline
\end{tabular}

Table 2: $\eta(*)$ and $q(*)$ equations

\begin{tabular}{|c|c|}
\hline$\eta(*)$ equations & $q(*)$ equations \\
\hline$A_{1}: \quad \eta^{\prime \prime}(x)-\eta^{2}=k(x) / 2$ & $B_{1}: \quad q^{\prime \prime}(x)+q^{2} / 2+k(x)=0$ \\
\hline$A_{2}: \quad \eta^{\prime \prime}(z)+c \eta^{\prime}(z)-\eta^{2}=k(z) / 2$ & $B_{2}: \quad q^{\prime \prime}(z)+c q^{\prime}(z)+q^{2} / 2+k(z)=0$ \\
\hline$A_{3}: \quad \eta^{\prime \prime}(\zeta)+\zeta \eta^{\prime}(\zeta) / 2-\eta^{2}+\eta=k(\zeta) / 2$ & $B_{3}: \quad q^{\prime \prime}(\zeta)+\zeta q^{\prime}(\zeta) / 2+q^{2}+q+k(\zeta)=0$ \\
\hline
\end{tabular}

2.2. Cubic Case. We will refer to the function $q_{2}(x, t)$ as $q(x, t)$ through all discussion of equation (1.2). The partial differential equation (1.2) has no (non-trivial) classical symmetries if $q(x, t)$ and $q_{1}(x, t)$ are free. Fix $q(x, t)$ as a solution to following partial differential equation

$$
\frac{\partial q}{\partial t}=\frac{\partial^{2} q}{\partial x^{2}}-\frac{1}{9} q^{3}+j(x, t) q+k(x, t),
$$

and fix $q_{1}(x, t)$ as a function of $q(x, t)$ as follows

$$
q_{1}(x, t)=j(x, t)-\frac{1}{3} q(x, t)^{2},
$$

where the functions $j(x, t)$ and $k(x, t)$ both satisfy partial differential equations, respectively,

$$
\begin{aligned}
& \left(c_{1}+\frac{c_{3} x}{2}\right) \frac{\partial j}{\partial x}+\left(c_{2}+c_{3} t\right) \frac{\partial j}{\partial t}+c_{3} j=0, \\
& \left(c_{1}+\frac{c_{3} x}{2}\right) \frac{\partial k}{\partial x}+\left(c_{2}+c_{3} t\right) \frac{\partial k}{\partial t}+\frac{3 c_{3} k}{2}=0 .
\end{aligned}
$$


Then, equation (1.2) is invariant under the three parameter Lie group of point symmetries with infinitesimal group operator (2.1) where

$$
\begin{aligned}
X(x, t, u) & =c_{1}+\frac{c_{3} x}{2}, \quad T(x, t, u)=c_{2}+c_{3} t, \\
U(x, t, u) & =\frac{1}{3}\left\{\left(c_{1}+\frac{c_{3} x}{2}\right) \frac{\partial q}{\partial x}+\left(c_{2}+c_{3} t\right) \frac{\partial q}{\partial t}\right\}-\frac{c_{3}}{2}\left(u-\frac{q}{3}\right),
\end{aligned}
$$

and $c_{1}, c_{2}, c_{3}$ are arbitrary parameters.

The partial differential equation (2.6) for $q(x, t)$ is also invariant under a three parameter Lie group of point symmetries if $j(x, t)$ and $k(x, t)$ satisfy equations $(2.8)$, with $c_{i} \mapsto c_{i+3}, i=1,2,3$. In this case

$$
X(x, t, q)=c_{4}+\frac{c_{6} x}{2}, \quad T(x, t, q)=c_{5}+c_{6} t, \quad Q(x, t, q)=\frac{-c_{6} q}{2},
$$

for arbitrary parameters $c_{4}, c_{5}, c_{6}$. Thus each of the similarity reductions of the independent variables valid for $u(x, t)$ are also valid for $q(x, t)$. These are the same reductions applied to the quadratic equation (1.1) above in Section 2.1. As such, we proceed in the same way here. Note that the dependent variable terms in equation (2.6) for $q(x, t)$ come directly from equation (1.2) with $u(x, t)=q(x, t) / 3$ and $q_{1}(x, t)$ given by $(2.7)$. The relationship between $u(x, t)$ and $q(x, t)$ is captured in each reduction of the dependent variable $u(x, t)$, as follows in Table 3.

Table 3: Summary of reductions of equation (1.2)

\begin{tabular}{cccccc}
\hline Case & $u(x, t)$ & $\eta(*)$ equation & $q(*)$ equation & $j(*)$ & $k(*)$ \\
\hline \hline$(11)$ & $q(x) / 3+\eta(x)$ & $C_{1}$ & $D_{1}$ & $j(x)$ & $k(x)$ \\
$(12)$ & $q(z) / 3+\eta(x)$ & $C_{1}$ & $D_{2}$ & constant & constant \\
$(13)$ & $q(\zeta) /\left(3 t^{1 / 2}\right)+\eta(x)$ & $C_{1}$ & $D_{3}$ & 0 & 0 \\
\hline$(21)$ & $q(x) / 3+\eta(z)$ & $C_{2}$ & $D_{1}$ & constant & constant \\
$(22)$ & $q(z) / 3+\eta(z)$ & $C_{2}$ & $D_{2}$ & $j(z)$ & $k(z)$ \\
$(23)$ & $q(\zeta) /\left(3 t^{1 / 2}\right)+\eta(z)$ & $C_{2}$ & $D_{3}$ & 0 & 0 \\
\hline$(31)$ & $q(x) / 3+\eta(x)$ & $C_{3}$ & $D_{1}$ & 0 & 0 \\
$(32)$ & $q(z) / 3+\eta(z)$ & $C_{3}$ & $D_{2}$ & 0 & 0 \\
$(33)$ & $(q(z) / 3+\eta(\zeta)) / t^{1 / 2}$ & $C_{3}$ & $D_{3}$ & $j(\zeta) / t$ & $k(\zeta) / t^{3 / 2}$ \\
\hline \hline
\end{tabular}

Table 4: $\eta(*)$ and $q(*)$ equations

\begin{tabular}{ll}
\hline \multicolumn{1}{c}{$\eta(*)$ equations } \\
\hline \hline$C_{1}:$ & $\eta^{\prime \prime}(x)-\eta^{3}+j(x) \eta=k(x) / 3$ \\
$C_{2}:$ & $\eta^{\prime \prime}(z)+c \eta^{\prime}(z)-\eta^{3}+j(z) \eta=k(z) / 3$ \\
$C_{3}:$ & $\eta^{\prime \prime}(\zeta)+\zeta \eta^{\prime}(\zeta) / 2-\eta^{3}+(2 j(\zeta)+1) \eta / 2=k(\zeta) / 3$ \\
\hline \multicolumn{1}{c}{$q(*)$ equations } \\
\hline \hline$D_{1}:$ & $q^{\prime \prime}(x)-q^{3} / 9+j(x) q+k(x)=0$ \\
$D_{2}:$ & $q^{\prime \prime}(z)+c q^{\prime}(z)-q^{3} / 9+j(z) q+k(z)=0$ \\
$D_{3}:$ & $q^{\prime \prime}(\zeta)+\zeta q^{\prime}(\zeta) / 2-q^{3} / 9+(2 j(\zeta)+1) q / 2+k(\zeta)=0$ \\
\hline \hline
\end{tabular}




\section{Exact Solutions}

3.1. Quadratic Case. In this section we give the transformation from each ordinary differential equation in Table 2 to the following equation

$$
y^{\prime \prime}(\lambda)=6 y^{2}+f(\lambda) \text {. }
$$

This is the canonical equation in the analysis of reductions of equation (1.1). Our goal is to solve equations of this form exactly. This equation is integrable if

$$
f(\lambda)=a \lambda+b,
$$

for $a$ and $b$ constant, and in such cases equation (3.1) can be solved exactly. For $a \neq 0$ equation (3.1) is equivalent to $\mathrm{P}_{\mathrm{I}}$, and the solution $y(\lambda)$ is a first Painlevé transcendent. For $a=0$ the solution to equation $(3.1)$ is $y(\lambda)=\wp\left(\lambda ;-2 b, g_{3}\right)$, where $\wp\left(\lambda ; g_{2}, g_{3}\right)$ is the Weierstrass elliptic function with invariants $g_{2}$ and $g_{3}$. Of the nine cases $(i j) i, j=1,2,3$, given in Table 1 , we find that only five are such that both the equation for $\eta$ and the equation for $q$ can be transformed to (3.1) with $f(\lambda)$ expressed in the form (3.2). For these five cases we provide exact solutions.

3.1.1. Transformations. Each set of equations in $\eta(*)$ and $q(*)$ given in Table 1 can be transformed to two equations of the form (3.1), in $y(\lambda)$ and $\hat{y}(\lambda)$. The transformations are

$$
\eta(*)=6 \gamma^{\prime}(*)^{2} y(\lambda)-\delta(*), \quad q(*)=-2\left(6 \gamma^{\prime}(*)^{2} \hat{y}(\lambda)-\delta(*)\right), \quad \lambda=\gamma(*),
$$

where $\delta(*)$, and $\gamma(*)$ are given below in Table 5 . The corresponding relations between the functions $k(*)$ and $f(\lambda)$ are

$$
k(*)=12 \gamma^{\prime}(*)^{4} f(\lambda)-\beta(*),
$$

where $\beta(*)$ is given in Table 5 , and $\lambda$ is given above.

Table 5: Terms in the transformations (3.3) and (3.4)

\begin{tabular}{ccccc}
\hline Equations & $*$ & $\delta(*)$ & $\beta(*)$ & $\gamma(*)$ \\
\hline \hline$A_{1} B_{1}$ & $x$ & 0 & 0 & $x$ \\
$A_{2} B_{2}$ & $z$ & $3 c^{2} / 25$ & $18 c^{4} / 625$ & $\exp (-c z / 5)$ \\
$A_{3} B_{3}$ & $\zeta$ & $\left(3 \zeta^{2}-40\right) / 100$ & $\left(3 \zeta^{4}+120 \zeta^{2}-600\right) / 625$ & $\sqrt{5 \pi} \operatorname{erf}(\zeta /(2 \sqrt{5}))$ \\
\hline \hline
\end{tabular}

3.1.2. Solutions. Comparing the conditions on $k(*)$ from Table 1 on the above forms we find that $f(\lambda)$ can only be linear in five cases. Exact solutions for these cases are given below in Table 6 . In this table $y(\lambda)$ and $\hat{y}(\lambda)$ are solutions to equation (3.1), related to $\eta(*)$ and $q(*)$ through $(3.3)$ where $\lambda=\gamma(*)$. The constant $b$ is fixed with $b=3 c^{4} / 625$, while $g_{2}, g_{3}$ and $\hat{g}_{3}$ are free in each case.

Table 6: Exact Solutions of Transformed Equations

\begin{tabular}{cccc}
\hline Case & Solution $y(\lambda)$ & Solution $\hat{y}(\lambda)$ & $\lambda$ \\
\hline \hline$(11)$ & $\mathrm{P}_{\mathrm{I}}$ or $\wp\left(\lambda ; g_{2}, g_{3}\right)$ & $\mathrm{P}_{\mathrm{I}}$ or $\wp\left(\lambda ; g_{2}, \hat{g}_{3}\right)$ & $x$ \\
$(12)$ & $\wp\left(x ; 0, g_{3}\right)$ & $\wp\left(\lambda ; b, \hat{g}_{3}\right)$ & $\gamma(z)$ \\
\hline$(21)$ & $\wp\left(\lambda ; b, g_{3}\right)$ & $\wp\left(x ; 0, \hat{g}_{3}\right)$ & $\gamma(z)$ \\
$(22)$ & $\mathrm{P}_{\mathrm{I}}$ or $\wp\left(\lambda ; g_{2}, g_{3}\right)$ & $\mathrm{P}_{\mathrm{I}}$ or $\wp\left(\lambda ; g_{2}, \hat{g}_{3}\right)$ & $\gamma(z)$ \\
\hline$(33)$ & $\mathrm{P}_{\mathrm{I}}$ or $\wp\left(\lambda ; g_{2}, g_{3}\right)$ & $\mathrm{P}_{\mathrm{I}}$ or $\wp\left(\lambda ; g_{2}, \hat{g}_{3}\right)$ & $\gamma(\zeta)$ \\
\hline \hline
\end{tabular}


Remark 3.1. Where the same reduction is applied to both $u(x, t)$ and $q(x, t)$, the solution $u(x, t)$ can be written concisely as a difference of first Painlevé transcendents or elliptic functions, as follows

$$
\begin{array}{lll}
\text { (11) } u(x, t)=6(y(\lambda)-\hat{y}(\lambda)), & \lambda=x, \\
(22) \quad u(x, t)=3 \gamma^{\prime}(z)^{2}(y(\lambda)-\hat{y}(\lambda)), & \lambda=\gamma(z), \\
(33) \quad u(x, t) & =3 \gamma^{\prime}(\zeta)^{2}(y(\lambda)-\hat{y}(\lambda)) /(2 t), & \lambda=\gamma(\zeta) .
\end{array}
$$

These solutions are constructed from the reductions given in Table 1 , the transformation (3.3) and finally the exact solutions $y(\lambda)$ and $\hat{y}(\lambda)$ where $\lambda=\gamma(*)$ is given in Table 5. For completeness, the corresponding form of $q(x, t)$ should also be given in each case, however this is easily calculated in the same way.

Remark 3.2. Where a different reduction is applied to $u(x, t)$ and $q(x, t)$, the solutions are

(12) $u(x, t)=6\left(y(x)-\gamma^{\prime}(\lambda)^{2} \hat{y}(\lambda)\right)+3 c^{2} / 25, q(x, t)=-6\left(2 \gamma^{\prime}(\lambda) \hat{y}(\lambda)-c^{2} / 25\right)$,

(21) $u(x, t)=-\left(6\left(y(x)-\gamma^{\prime}(\lambda)^{2} \hat{y}(\lambda)\right)+3 c^{2} / 25\right), \quad q(x, t)=-12 \hat{y}(x)$,

where $\lambda=\gamma(z)$ in each case. Here it is useful to specify $q(x, t)$ to distinguish the two cases.

3.2. Cubic Case. The canonical equation in the analysis of reductions of equation $(1.2)$ is

$$
y^{\prime \prime}(\lambda)=2 y^{3}+g(\lambda) y+h(\lambda) .
$$

Our goal is to solve equations of this form exactly. This equation is integrable if

$$
g(\lambda)=a \lambda+b, \quad h(\lambda)=d,
$$

for $a, b$, and $d$ constant, and in such cases equation (3.5) can be solved exactly. For $a \neq 0$ equation (3.5) is equivalent to $\mathrm{P}_{\mathrm{II}}$, and the solution $y(\lambda)$ is a second Painlevé transcendent. For $a=0$ the solution can be expressed in terms of Jacobi elliptic functions.

3.2.1. Transformations. Each set of equations for $\eta(*)$ and $q(*)$ given in Table 3, can be transformed to two equations of the form (3.5), in $y(\lambda)$ and $\hat{y}(\lambda)$. The transformations are

$$
\eta(*)=\sqrt{2} \gamma^{\prime}(*) y(\lambda), \quad q(*)=3 \sqrt{2} \gamma^{\prime}(*) \hat{y}(\lambda), \quad \lambda=\gamma(*),
$$

where $\gamma(*)$ is given below in Table 7 . The corresponding relations between the function $j(*)$ and $g(\lambda)$, and $k(*)$ and $h(\lambda)$ are

$$
j(*)=-\gamma^{\prime}(*)^{2} g(\lambda)+\beta(*), \quad k(*)= \pm 3 \sqrt{2} \gamma^{\prime}(*)^{3} h(\lambda),
$$

where $\beta(*)$ is also given in Table 7 and $\lambda$ is given above. The \pm in the $k(*)$ expression refers to the $\eta(*)$, and $q(*)$ equations, respectively.

Table 7: Terms in the transformations (3.7) and (3.8)

\begin{tabular}{cccc}
\hline Equations & $*$ & $\beta(*)$ & $\gamma(*)$ \\
\hline \hline$C_{1} D_{1}$ & $x$ & 0 & $x$ \\
$C_{2} D_{2}$ & $z$ & $2 c^{2} / 9$ & $-3 \exp (-c z / 3) / c$ \\
$C_{3} D_{3}$ & $\zeta$ & $\left(\zeta^{2}-6\right) / 18$ & $\sqrt{3 \pi} \operatorname{erf}(\zeta /(2 \sqrt{3}))$ \\
\hline \hline
\end{tabular}


3.2.2. Solutions. Comparing the conditions on $k(*)$ and $j(*)$ from Table 3 on the above forms we find that $g(\lambda)$ is linear and $h(\lambda)$ is constant only in five cases. These are the same five cases as in the quadratic case. Before presenting the exact solutions we note the following: 1 . The second Painlevé equation contains a parameter $\alpha$. When the solutions $y(*)$ and $\hat{y}(*)$ are expressed in terms of second Painlevé transcendents, this is for different parameters: $\alpha$ and $-\alpha$ respectively. We denote these $y(*)=\mathrm{P}_{\mathrm{II}}(* ; \alpha)$ and $\hat{y}(*)=\mathrm{P}_{\mathrm{II}}(* ;-\alpha) ; 2$. There is no canonical Jacobi elliptic differential equation corresponding to (3.5) with $g(\lambda)$ and $h(\lambda)$ both constant. Rather, the exact expression of the solution in terms of the Jacobi elliptic functions depends on the value these constants take (see [2]). Thus for equations of the form

$$
y^{\prime \prime}(*)=2 y^{3}+b y+d, \quad b, d \quad \text { arbitrary, }
$$

we denote the solution as $y(*)=\operatorname{JE}(* ; b, d)$. In the following table $b$ and $d$ are free constants in each case.

Table 8: Exact Solutions of Transformed Equations

\begin{tabular}{cccc}
\hline Case & Solution $y(\lambda)$ & Solution $\hat{y}(\lambda)$ & $\lambda$ \\
\hline \hline$(11)$ & $\mathrm{P}_{\mathrm{II}}(\lambda ; \alpha)$ or $\mathrm{JE}(\lambda ; b, d)$ & $\mathrm{P}_{\mathrm{II}}(\lambda ;-\alpha)$ or $\mathrm{JE}(\lambda ; b,-d)$ & $x$ \\
$(12)$ & $\mathrm{JE}\left(x ;-2 c^{2} / 9,0\right)$ & $\mathrm{JE}(\lambda ; 0,0)$ & $\gamma(z)$ \\
\hline$(21)$ & $\mathrm{JE}(\lambda ; 0,0)$ & $\mathrm{JE}\left(x ;-2 c^{2} / 9,0\right)$ & $\gamma(z)$ \\
$(22)$ & $\mathrm{P}_{\mathrm{II}}(\lambda ; \alpha)$ or $\mathrm{JE}(\lambda ; b, d)$ & $\mathrm{P}_{\mathrm{II}}(\lambda ; \alpha)$ or $\mathrm{JE}(\lambda ; b,-d)$ & $\gamma(z)$ \\
\hline$(33)$ & $\mathrm{P}_{\mathrm{II}}(\lambda ; \alpha)$ or $\mathrm{JE}(\lambda ; b, d)$ & $\mathrm{P}_{\mathrm{II}}(\lambda ; \alpha)$ or $\mathrm{JE}(\lambda ; b,-d)$ & $\gamma(\zeta)$ \\
\hline \hline
\end{tabular}

\section{Discussion}

In this paper we have found five exact solutions to each of the variable-coefficient reaction diffusion equations (1.1) and (1.2). These were constructed by forcing the reduced ordinary differential equations associated with (1.1) and (1.2) to be integrable. The symmetry analysis also revealed solutions given in terms of nonintegrable ordinary differential equations, cases (13), (23), (31) and (32), which remain to be explored. The exact solutions we have found are given in terms of Painlevé transcendents or elliptic functions. The initial conditions for the Painlevé transcendents, and the parameter for $\mathrm{P}_{\mathrm{II}}$, have not been specified here. Similarly the invariants $g_{2}, g_{3}$ in the Weierstrass elliptic function solutions, and parameters in the Jacobi elliptic function solutions, have been left as arbitrary in many cases. The specific dynamics of the solutions can be investigated for each choice of these inputs that are of interest.

\section{REFERENCES}

1. Mark J. Ablowitz and Anthony Zeppetella, Explicit solutions of Fisher's equation for a special wave speed, Bull. Math. Biol. 41 (1979), no. 6, 835-840. MR MR639998 (83g:35082)

2. Milton Abramowitz and Irene A. Stegun, Handbook of mathematical functions with formulas, graphs, and mathematical tables, National Bureau of Standards Applied Mathematics Series, vol. 55, For sale by the Superintendent of Documents, U.S. Government Printing Office, Washington, D.C., 1964. MR MR0167642 (29 \#4914)

3. George W. Bluman and Stephen C. Anco, Symmetry and integration methods for differential equations, Applied Mathematical Sciences, vol. 154, Springer-Verlag, New York, 2002. MR MR1914342 (2003i:34090) 
4. B. H. Bradshaw-Hajek, M.P. Edwards, P. Broadbridge, and G.H. Williams, Nonclassical symmetry solutions for reaction-diffusion equations with explicit spatial dependence, Preprint submitted to Elsevier Science, 8 August 2006.

5. Robert Stephen Cantrell and Chris Cosner, Spatial ecology via reaction-diffusion equations, Wiley Series in Mathematical and Computational Biology, John Wiley \& Sons Ltd., Chichester, 2003. MR MR2191264 (2007a:92069)

6. Peter A. Clarkson and Elizabeth L. Mansfield, Symmetry reductions and exact solutions of a class of nonlinear heat equations, Phys. D 70 (1994), no. 3, 250-288. MR MR1258270 (95b:35092)

7. M. D. Kruskal, N. Joshi, and R. Halburd, Analytic and asymptotic methods for nonlinear singularity analysis: a review and extensions of tests for the Painlevé property, Integrability of nonlinear systems (Pondicherry, 1996), Lecture Notes in Phys., vol. 495, Springer, Berlin, 1997, pp. 171-205. MR MR1636294 (99g:34031)

8. J. D. Murray, Mathematical biology. I, third ed., Interdisciplinary Applied Mathematics, vol. 17, Springer-Verlag, New York, 2002, An introduction. MR MR1908418 (2004b:92003)

9. Akira Okubo and Simon A. Levin, Diffusion and ecological problems: modern perspectives, second ed., Interdisciplinary Applied Mathematics, vol. 14, Springer-Verlag, New York, 2001. MR MR1895041 (2003a:92025)

School of Mathematics and Statistics F07, University of Sydney, NSW 2006

E-mail address: nalini@maths.usyd.edu.au

School of Mathematics and Statistics F07, University of Sydney, NSW 2006

E-mail address: teganm@maths.usyd.edu.au 